\title{
The origin of the molecular emission around the southern hemisphere Re 4 IRS - HH 188 region
}

\author{
J. M. Girart ${ }^{1}$ and S. Viti ${ }^{2}$ \\ 1 Institut de Ciències de l'Espai (CSIC- IEEC), Campus UAB - Facultat de Ciències, Torre C5 - parell 2, 08193 Bellaterra, \\ Catalunya, Spain \\ e-mail: girart@ieec.cat \\ 2 Department of Physics and Astronomy, University College London, London WC1E 6BT, UK
}

Received 24 August 2006 / Accepted 10 May 2007

\begin{abstract}
Aims. We present SEST observations of the molecular environment ahead of the southern Herbig-Haro object 188 (HH 188), associated with the low-mass protostar Re 4 IRS. We have also used the SuperCosmos $\mathrm{H} \alpha$ survey to search for $\mathrm{H} \alpha$ emission associated with the Re 4 IRS - HH 188 region. The aim of the present work is to study the properties of the molecular gas and to better characterize this southern star forming region.

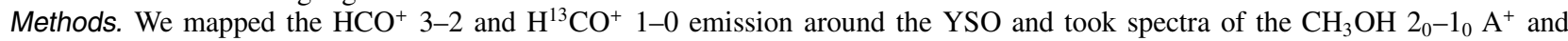
$2_{-1}-1_{-1}$ E and SO $65_{5}-5_{4}$ towards the central source. Column densities are derived and different scenarios are considered to explain the origin of the molecular emission.

Results. $\mathrm{HCO}^{+}$arises from a relatively compact region around the YSO; however, its peak emission is displaced to the south following the outflow direction. Our chemical analysis indicates that a plausible scenario is that most of the emission arises from the cold, illuminated dense gas ahead of the $\mathrm{HH} 188$ object. We have also found that $\mathrm{HH} 188$, a high excitation object, seems to be part of a parsec scale and highly collimated HH system. Re 4 IRS is probably a binary protostellar system, in the late Class 0 or Class I phase. One of the protostars, invisible in the near-IR, seems to power the HH 188 system.
\end{abstract}

Key words. ISM: individual objects: HH 188 - ISM: abundances - ISM: clouds - ISM: molecules - radio lines: ISM stars: formation

\section{Introduction}

Re 4 is an optical reflection nebula, also classified as a cometary globule (Reipurth 1981), in the dark cloud listed as No. 109 by Sandqvist (1977). This cloud is located within the Gum Nebula at a distance of $\sim 450 \mathrm{pc}$. Spectroscopical observations in the optical of the nebula (Graham 1986) shows a very red continuum emission and some absorption ( $\mathrm{Na}$ I D) and emission lines, mainly [SII], $\mathrm{H} \alpha$, [OI]. The emission line strengths resemble those of the low-excitation $\mathrm{HH}$ objects. Re 4 is associated with a low-mass protostar. Emission associated with the protostar or its dense circumstellar environment is detected from near-IR (2MASS J08205824-4934456), mid and far IR (IRAS 08194-4925) through to millimeter wavelengths (Graham 1986; Reipurth et al. 1993; Dent et al. 1998). The protostar (hereafter Re 4 IRS) has a bolometric luminosity of $\sim 30 L_{\odot}$ (Cohen \& Schwartz 1987). There is a star located only 3"' 1 northeast of Re 4 and detected also in the near-IR (2MASS J08205862-4934443). Its optical spectroscopic profile suggests that is a K5 V foreground star (Graham 1986). Re 4 IRS is associated with a Herbig-Haro object, HH 188, which appears as a series of few knots extending about $10^{\prime \prime}$ south of the Re 4 nebulosity (Graham 1986). The optical spectra of these knots show emission in [SII], $\mathrm{H} \alpha$, [OI] and [NaII].

VLT near-IR observations show that the Re 4 reflection nebulosity extends further away from the protostar with a S-like morphology, blue in the south, red in the north, probably indicating that the northern lobe is further from us and more embedded in the parent cloud (Zinnecker et al. 1999). This peculiar shape is possibly tracing excavated cavities by the outflow associated with HH 188. The VLT images show hints of what appear to be a binary jet emanating from the obscured centre, with an offset angle between the two jets of 5-10 (Zinnecker et al. 1999).

In this paper we present SEST observations of the extended molecular emission around Re 4 IRS and HH 188 as well as complementary data taken from public optical/near-IR data. In Sect. 2 we describe the observations. In Sect. 3 we describe the results of the observations and we analyze the molecular emission around Re 4 and HH 188. In Sect. 4 we describe the search for possible new $\mathrm{HH}$ objects associated with $\mathrm{HH} 188$ by using the $\mathrm{H} \alpha$ SuperCosmos survey. In Sect. 5 we discuss the properties of Re 4 IRS, HH 188 and its association with a larger HH system. We also discuss the different possibilities for the origin of the molecular emission. In Sect. 6 we summarize our findings.

\section{Observations}

The observations were carried out in January 10 and 11, 2003 with the SEST $15 \mathrm{~m}$ radio telescope at La Silla (Chile). We used the dual receiver capability of the telescope to observe two molecular lines simultaneously, one at $3 \mathrm{~mm}$ and the other at $1 \mathrm{~mm}$ : hence we observed simultaneously the $\mathrm{HCO}^{+} 3-2$ and $\mathrm{H}^{13} \mathrm{CO}^{+} 1-0$ as well as the SO $6_{5}-5_{4}$ and $\mathrm{CH}_{3} \mathrm{OH} 2_{0}-1_{0} \mathrm{~A}^{+}$. We used the High Resolution Acousto-Optic Spectrometer, which provided a spectral resolution of $\sim 0.14$ and $0.05 \mathrm{~km} \mathrm{~s}^{-1}$ at 3 and $1 \mathrm{~mm}$, respectively (the $1 \mathrm{~mm}$ data was smoothed to a resolution of $\sim 0.3 \mathrm{~km} \mathrm{~s}^{-1}$ to increase the signal-to-noise ratio). 
Table 1. Lines detected with the SEST telescope.

\begin{tabular}{lccccc}
\hline \hline & & $\begin{array}{c}v \\
(\mathrm{GHz})\end{array}$ & $\begin{array}{l}\text { Beam } \\
(F W H M)\end{array}$ & $\begin{array}{c}\Delta v \\
\left(\mathrm{~km} \mathrm{~s}^{-1}\right)\end{array}$ & $\begin{array}{r}T_{\mathrm{mb}} \\
\mathrm{mK}\end{array}$ \\
\hline $\mathrm{H}^{13} \mathrm{CO}^{+}$ & $1-0$ & 86.7543 & $57^{\prime \prime}$ & 0.15 & 46 \\
$\mathrm{CH}_{3} \mathrm{OH}$ & $2{ }_{0}-1_{0} \mathrm{~A}^{+a}$ & 96.7414 & $51^{\prime \prime}$ & 0.13 & 38 \\
$\mathrm{SO}$ & $6_{5}-5_{4}$ & 219.9494 & $24^{\prime \prime}$ & 0.12 & 54 \\
$\mathrm{HCO}^{+}$ & $3-2$ & 267.5576 & $20^{\prime \prime}$ & 0.19 & 110 \\
\hline
\end{tabular}

${ }^{a}$ These spectra included also the $2_{-1}-1_{-1}$ E line.

The observations were taken using the frequency switching mode, which gives a bandwidth of 120 and $30 \mathrm{~km} \mathrm{~s}^{-1}$ at 3 and $1 \mathrm{~mm}$, respectively. For the $\mathrm{HCO}^{+}$and $\mathrm{H}^{13} \mathrm{CO}^{+}$lines we did a grid of 26 points, with a cellsize of $20^{\prime \prime}$, covering an area of 1 1.5 $\times 1$ 1.5 around $\mathrm{Re} 4 \mathrm{IRS}$. The $\mathrm{SO}$ and $\mathrm{CH}_{3} \mathrm{OH}$ were only observed towards the IR source. Table 1 lists the lines observed, their frequencies, the SEST FWHM (full width at half maximum) beam size for these frequencies, the spectral resolution of the data and the rms noise in units of main beam temperature. Average system temperatures during the observations were about $150,180,170,600 \mathrm{~K}$ at 86, 97, 220 and $267 \mathrm{GHz}$, respectively. Main beam efficiency at these same frequencies were $0.75,0.72,0.52$ and 0.42 .

\section{Results and analysis}

Figure 1 shows the integrated emission map of the $\mathrm{HCO}^{+} 3-2$ and $\mathrm{H}^{13} \mathrm{CO}^{+} 1-0$ transitions. Figure 2 shows the spectra of the $\mathrm{HCO}^{+}$isotopes as well as those of the $\mathrm{SO}$ and $\mathrm{CH}_{3} \mathrm{OH}$ lines. Table 5 shows the results of Gaussian fits to the spectra at four different positions. The emission appears to be relatively compact around $\mathrm{Re} 4 \mathrm{IRS}$. From the $\mathrm{HCO}^{+} 3-2$ map, which has the highest angular resolution $\left(\sim 20^{\prime \prime}\right)$, the FWHM contour has a diameter of $\sim 50^{\prime \prime}$, which implies a deconvolved size of $\sim 45^{\prime \prime}$ or $0.09 \mathrm{pc}$ (assuming that the emission arises from a single Gaussian component). The peak of the $\mathrm{HCO}^{+} 3-2$ emission is near $\mathrm{HH} 188$, approximately at the $+5^{\prime \prime},-12^{\prime \prime}$ offset position, i.e. $\alpha(\mathrm{J} 2000)=08^{\mathrm{h}} 20^{\mathrm{m}} 59^{\mathrm{s}} .2$ and $\delta(\mathrm{J} 2000)=-49^{\circ} 35^{\prime} 00^{\prime \prime}$.

From the spectra (see Fig. 3 and Table 5) one can see that the lines are narrow, with a width of $\Delta v \simeq 0.7$ to $0.8 \mathrm{~km} \mathrm{~s}^{-1}$ for the $\mathrm{H}^{13} \mathrm{CO}^{+}$line, which is optically thin, and that there are no significant velocity gradients along the region $\left(\lesssim 0.4 \mathrm{~km} \mathrm{~s}^{-1}\right)$. The $\mathrm{HCO}^{+} 3-2$ spectra do not show self-absorption. Redshifted self-absorption in optically thick lines can be a signature of infall motions (e.g., Zhou et al. 1993; Rawlings \& Yates 2001).

The column densities were derived in the following manner: for the $\mathrm{SO}$ and $\mathrm{CH}_{3} \mathrm{OH}$ we assumed optically thin emission and an excitation temperature of $10 \mathrm{~K}$. For the $\mathrm{HCO}^{+}$, we first convolved the $\mathrm{HCO}^{+} 3-2$ emission with a Gaussian to obtain a final beam size of $57^{\prime \prime}$, the angular resolution of the $\mathrm{H}^{13} \mathrm{CO}^{+}$spectra. Then, the $\mathrm{HCO}^{+} 3-2$ and $\mathrm{H}^{13} \mathrm{CO}^{+} 1-0$ optical depth were derived from the line ratio of the $\mathrm{H}^{13} \mathrm{CO}^{+}$to the convolved $\mathrm{HCO}^{+}$spectra at the positions given in Table 5 and assuming a ${ }^{12} \mathrm{C}$ to ${ }^{13} \mathrm{C}$ ratio of 63 (Langer \& Penzias 1993). An excitation temperature of $7 \mathrm{~K}$ was adopted for the $\mathrm{HCO}^{+}$lines, which is the typical value found for this molecule in the illuminated condensations ahead of $\mathrm{HH}$ objects (Girart et al. 2002; Viti et al. 2006). We find that the $\mathrm{H}^{13} \mathrm{CO}^{+}$line is optically thin, $\tau \sim 0.5$, whereas the $\mathrm{HCO}^{+}$line optically thick, $\tau \sim 20$. The column density of the $\mathrm{HCO}^{+}$is derived from both the original and the convolved $\mathrm{HCO}^{+} 3-2$ spectra, assuming LTE for the aforementioned excitation temperature and correcting for the optical depth (see Table 2).

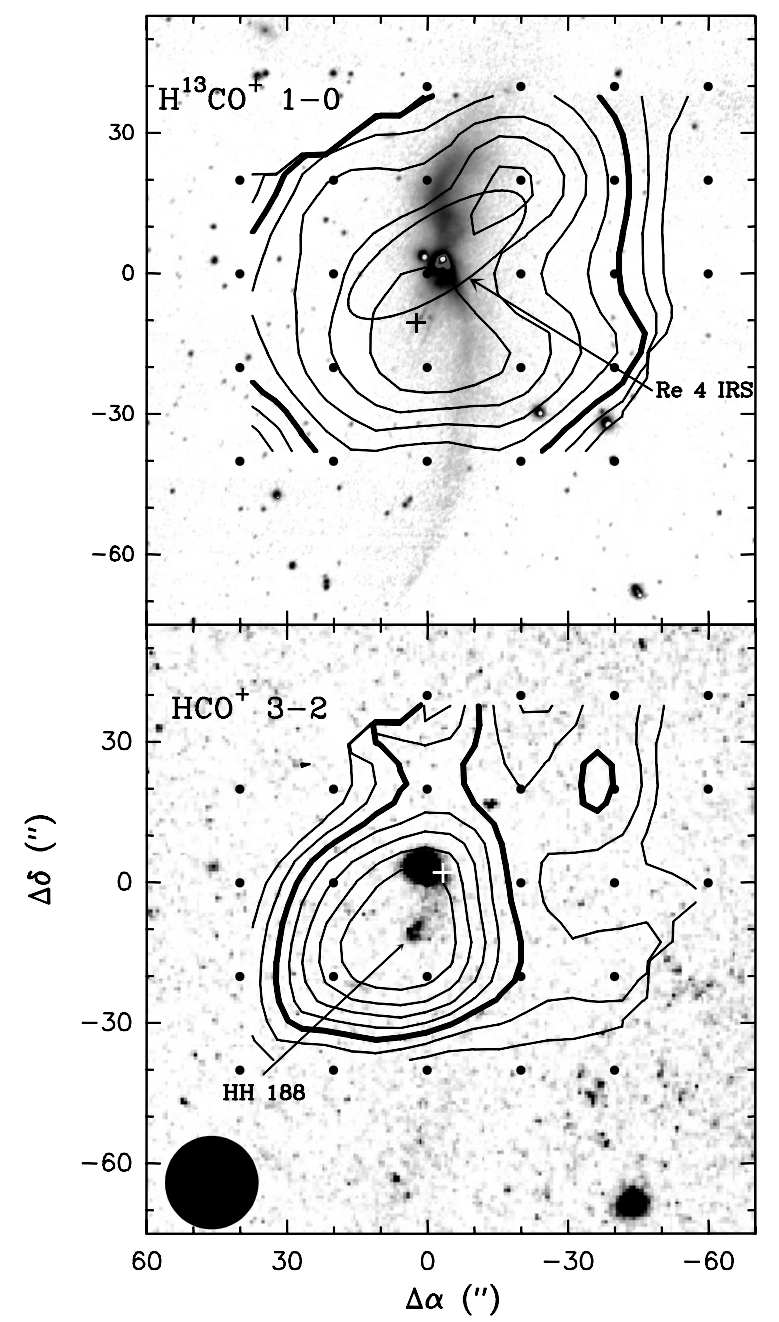

Fig. 1. Top panel: superposition of the $\mathrm{K}_{\mathrm{s}}$ image (from Zinnecker et al. 1999) with the SEST contour map of the $\mathrm{H}^{13} \mathrm{CO}^{+} 1-0$ emission towards the HH 188 region. Bottom panel: superposition of the $\mathrm{H} \alpha$ image (from the AAO/UKST SuperCOSMOS H $\alpha$ survey, Parker et al. 2005) with the SEST contour map of the $\mathrm{HCO}^{+} 3-2$ emission. For both lines the emission was averaged over the $2.75-4.80 \mathrm{~km} \mathrm{~s}^{-1} v_{\mathrm{LSR}}$ interval. The contour levels are 0.15 to 0.5 with $0.05 \mathrm{~K} \mathrm{~km} \mathrm{~s}^{-1}$ steps for $\mathrm{H}^{13} \mathrm{CO}^{+} 1-0$, and 0.3 to 1.0 with $0.1 \mathrm{~K} \mathrm{~km} \mathrm{~s}^{-1}$ steps for $\mathrm{HCO}^{+} 3-2$. The center of the maps corresponds to the position $\alpha(\mathrm{J} 2000)=08^{\mathrm{h}} 20^{\mathrm{m}} 58.59$ and $\delta(\mathrm{J} 2000)=$ $-49^{\circ} 34^{\prime} 47^{\prime \prime} 8$. Small filled circles show the observed positions. The ellipsoid shows the position uncertainty of IRAS 08194-4925. The black cross in the top panel shows the position of HH 188. The white cross in the bottom panel shows the position of Re 4 IRS derived from the VLT $\mathrm{K}_{\mathrm{s}}$ image. The filled circle at the bottom-left corner of the bottom panel shows the beam size of the $\mathrm{HCO}^{+} 3-2$ map, $20^{\prime \prime}$.

In order to derive the fractional abundances we first estimate the total molecular hydrogen column density from the dust emission. We used the dust measured with the SEST at $1.3 \mathrm{~mm}$ (therefore at a similar angular resolution of our $\mathrm{SO}$ and $\mathrm{HCO}^{+}$ observations) by Reipurth et al. (1993), $S_{1.3 \mathrm{~mm}}=0.27 \mathrm{Jy}$. By adopting a dust opacity of $\kappa_{1.3 \mathrm{~mm}}=1.0 \mathrm{~cm}^{2} \mathrm{~g}^{-1}$ (Ossenkopf \& Henning 1994), a gas-to-mass ratio of 100 and a dust temperature of $35 \mathrm{~K}$ (Reipurth et al. 1993), the beam averaged column density of the molecular hydrogen is $2.6 \times 10^{22} \mathrm{~cm}^{-2}$. Thus, the fractional abundances of $\mathrm{SO}$ and $\mathrm{HCO}^{+}$are $X[\mathrm{SO}]=8 \times 10^{-10}$ and $X\left[\mathrm{HCO}^{+}\right]=4 \times 10^{-9}$. For the $\mathrm{CH}_{3} \mathrm{OH}$, we first assume that the $\mathrm{CH}_{3} \mathrm{OH}$ and $\mathrm{HCO}^{+}$trace the same gas. In this case, the column density ratio of these two species will be the same at $57^{\prime \prime}$ 

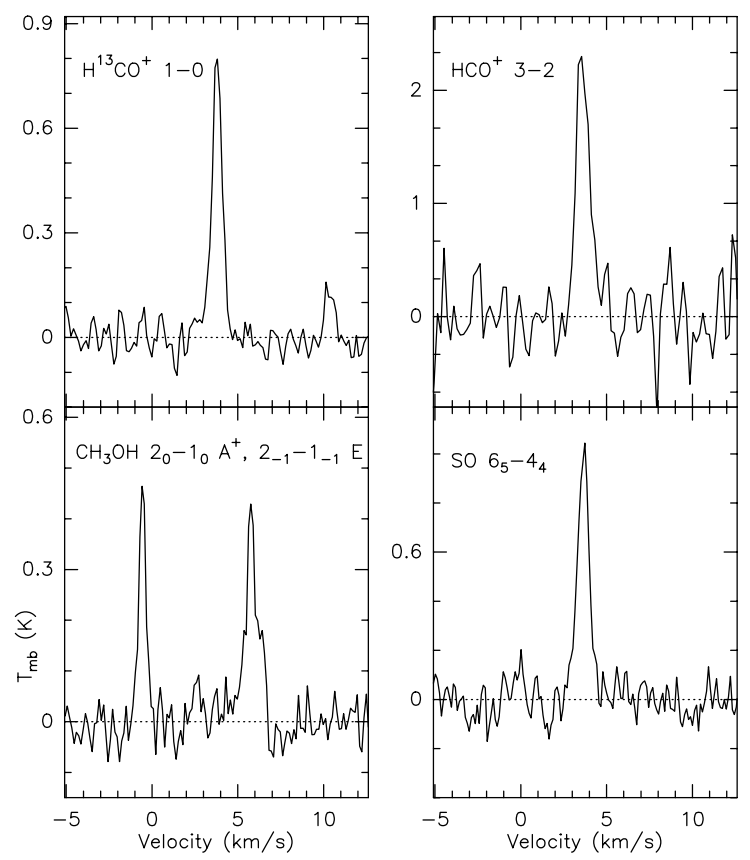

Fig. 2. SEST spectra at the $\left(0^{\prime \prime}, 0^{\prime \prime}\right)$ of the $\mathrm{HCO}^{+} 3-2, \mathrm{H}^{13} \mathrm{CO}^{+} 1-0$, $\mathrm{CH}_{3} \mathrm{OH} 2_{0}-1_{0} \mathrm{~A}^{+}$and $2_{-1}-1_{-1} \mathrm{E}$, and $\mathrm{SO}_{5}-5_{4}$ lines.

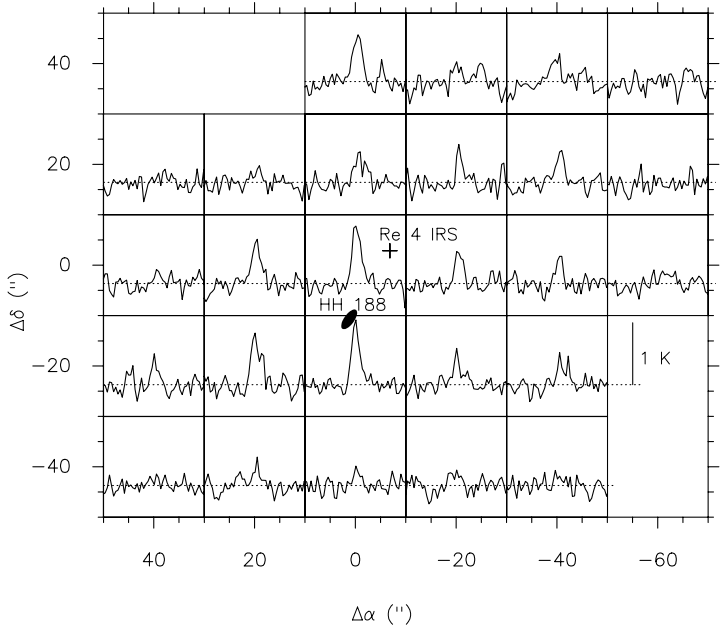

Fig. 3. Spectra of the $\mathrm{HCO}^{+} 3-2$ towards $\mathrm{HH} 188$. The velocity range for all the spectra is from -1 to $8 \mathrm{~km} \mathrm{~s}^{-1}$, and the main brightness temperature range (abscissa axis) is from -0.5 to $1.15 \mathrm{~K}$. The scale for $1 \mathrm{~K}$ is shown as a bar (bottom right). The cross and the filled ellipsoid show the position of Re 4 IRS and HH 188, respectively. The center of the map corresponds to the position $\alpha(\mathrm{J} 2000)=08^{\mathrm{h}} 20^{\mathrm{m}} 58^{\mathrm{s}} .642$ and $\delta(\mathrm{J} 2000)=-49^{\circ} 34^{\prime} 47^{\prime \prime} .57$.

(the angular resolution of the $\mathrm{CH}_{3} \mathrm{OH}$ spectra) and at $20^{\prime \prime}$. From here, the derived fractional abundance is $X\left[\mathrm{CH}_{3} \mathrm{OH}\right]=3 \times 10^{-9}$.

\section{Inspection of the $\mathrm{H} \alpha$ SuperCosmos survey}

We have examined the $\mathrm{H} \alpha$ SuperCosmos survey in a region of $20^{\prime} \times 20^{\prime}$ around HH 188 in order to check for possible HH-like objects that may be associated to this region. Figure 4 shows the narrow band image centered at the $\mathrm{H} \alpha$ image and the red broad band continuum image. We have found several knots of pure $\mathrm{H} \alpha$ emission. Table 3 shows the position, distance and the position angle with respect to Re 4 IRS (see Sect. 5.1). Around Re 4 IRS there is a compact (a size of $\sim 3^{\prime \prime}$ ) counterpart of
Table 2. Column density and fractional abundance ${ }^{a}$ with respect to molecular hydrogen.

\begin{tabular}{lccc}
\hline \hline Molecule & $N[\mathrm{~mol}]^{b}$ & $N[\mathrm{~mol}]^{c}$ & $X[\mathrm{~mol}]$ \\
\hline$\left(0^{\prime \prime}, 0^{\prime \prime}\right)$ & & & \\
$\mathrm{HCO}^{+}$ & $5.6(13)$ & $1.1(14)$ & $4(-9)$ \\
$\mathrm{CH}_{3} \mathrm{OH}$ & $4.3(13)$ & $\ldots$ & $3(-9)$ \\
$\mathrm{SO}$ & $\ldots$ & $2.1(13)$ & $8(-10)$ \\
$\left(0^{\prime \prime},-20^{\prime \prime}\right)$ & & & \\
$\mathrm{HCO}^{+}$ & $5.7(13)$ & $1.2(14)$ & $\ldots$ \\
$\left(20^{\prime \prime},-20^{\prime \prime}\right)$ & & & \\
$\mathrm{HCO}^{+}$ & $4.9(13)$ & $1.2(14)$ & $\ldots$ \\
$\left(20^{\prime \prime}, 0^{\prime \prime}\right)$ & & & \\
$\mathrm{HCO}^{+}$ & $4.4(13)$ & $7.5(13)$ & $\ldots$ \\
\hline
\end{tabular}

${ }^{a} a(b)$ stands for $a \times 10^{b}$. Column density units in $\mathrm{cm}^{-3}$. Abundances derived assuming $N\left(\mathrm{H}_{2}\right)=2.6 \times 10^{22} \mathrm{~cm}^{-3} \cdot{ }^{b}$ Beam averaged column density for a beam of $57^{\prime \prime} .{ }^{c}$ Beam averaged column density for a beam of $20^{\prime \prime}$.
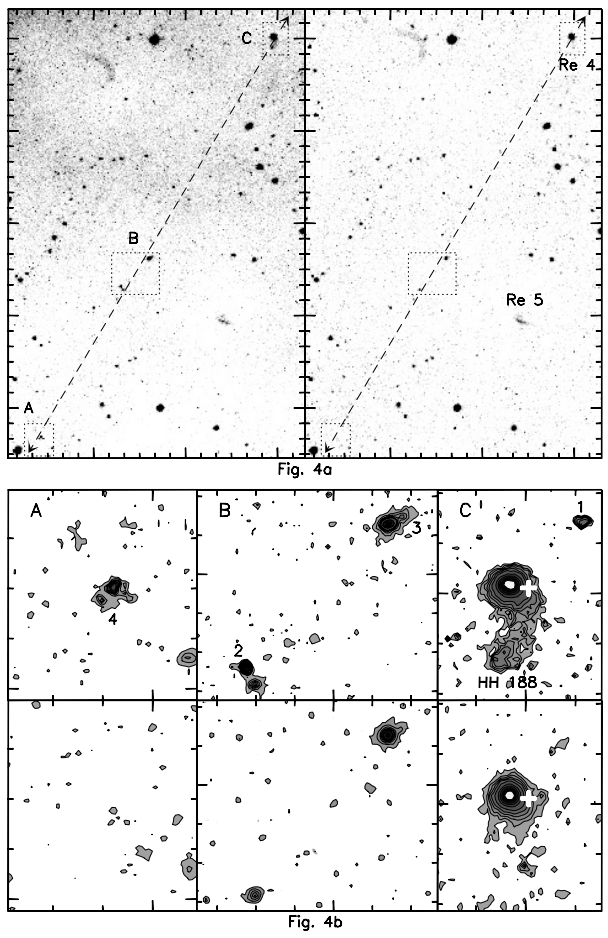

Fig. 4. (a) Narrow-band $\mathrm{H} \alpha$ (left panel) and contemporaneous matching broad-band Short-Red (right panel) images of the HH 188 region. The images are obtained from the AAO/UKST SuperCOSMOS H $\alpha$ survey (Parker et al. 2005). The dashed arrowed line shows the direction of the possible HH 188 outflow system. The small dotted rectangles show the enlarged regions shown in the bottom panels. (b) Narrow-band $\mathrm{H} \alpha$ (top panels) and broad-band Short-Red (bottom panels) images of the selected regions in (a), where pure $\mathrm{H} \alpha$ is detected along the direction of the HH 188 outflow system. The thick cross in the right panels indicate the position of the nea-IR peak emission associated with Re 4 IRS.

HH 188 northwest of the infrared source. This knot and HH 188 are separated by $31^{\prime \prime} .6$ with a position angle of $148.9^{\circ}$. Southeast of $\mathrm{HH} 188$ we have found three other relatively compact knots $\left(\sim 3^{\prime \prime}\right)$ of pure $\mathrm{H} \alpha$ emission. Object 2 is close to a star (4".5). However, an inspection of the 2MASS catalogue shows that this star has colors, $J-H=0.64$ and $H-K=0.32$, indicating that it is a main sequence star. This suggests that the $\mathrm{H} \alpha$ emission is not related with the star. The five $\mathrm{H} \alpha$ objects, including HH 188, appear very well aligned (see Fig. 4) with a position 
Table 3. Tentative identification of $\mathrm{HH}$ objects.

\begin{tabular}{cccrcr}
\hline \hline $\begin{array}{c}\text { Object } \\
\text { Num. }\end{array}$ & $\begin{array}{c}\alpha(\mathbf{J} 2000) \\
\left(08^{\mathrm{h}}\right)\end{array}$ & $\begin{array}{c}\delta(\mathrm{J} 2000) \\
\left(-49^{\circ}\right)\end{array}$ & $\begin{array}{c}\text { Distance } \\
\left({ }^{\prime}\right)\end{array}$ & $\begin{array}{c}\mathrm{PA}^{a} \\
\left({ }^{\circ}\right)\end{array}$ & Notes \\
\hline 1 & $20^{\mathrm{m}} 57^{\mathrm{s}} .10$ & $34^{\prime} 31^{\prime \prime} .2$ & 15.8 & 148.7 & \\
2 & $20^{\mathrm{m}} 58^{\mathrm{s}} .78$ & $34^{\prime} 58^{\prime \prime} 1$ & 15.8 & 148.7 HH 188 \\
3 & $21^{\mathrm{m}} 14.68$ & $39^{\prime} 32^{\prime \prime} .5$ & 333.3 & 149.7 & \\
4 & $21^{\mathrm{m}} 18.80$ & $40^{\prime} 11^{\prime \prime} .6$ & 385.0 & 148.2 & \\
5 & $21^{\mathrm{m}} 29^{\mathrm{s}} .47$ & $43^{\prime} 28^{\prime \prime} .5$ & 607.0 & 149.7 & \\
\hline
\end{tabular}

a Distance and position angle with respect to the driving source, Re 4 IRS, which is assumed to lie between HH-like object 1 and HH 188 (see Sect. 5.1)

angle of $\simeq 149^{\circ}$. We checked the 2 MASS catalogue and neither of these sources have emission in the near-IR. The lack of red continuum and near-IR emission suggests that these objects are probably $\mathrm{HH}$ objects, apparently forming part of the same system and associated with HH 188.

\section{Discussion}

\subsection{Re 4 IRS and the HH 188 system}

Figure 5 shows the spectral energy distribution (SED) of the driving source of $\mathrm{HH} 188, \mathrm{Re} 4$ IRS.

Whitney et al. (2003) carried out detailed radiative transfer models of protostellar envelopes, showing the expected SED and the near-IR morphology for different inclination angles and evolutionary stages. By using the Re 4 IRS's SED and the color composite $J H K$ image obtained by Zinnecker et al. (1999) we found that this YSO is possibly a late Class 0 or Class I object, with an inclination angle of roughly $\sim 30^{\circ}$. Graham \& Heyer (1989) pointed out that the near-IR emission associated with Re 4 IRS has a cometary structure (see Fig. 6), which indicates that is tracing the scattered stellar light arising from the cavity created by the outflow. Comparing the images at $R, J, H, K \mathrm{~s}$, the cometary structure appears to be displaced to the northwest at increasing wavelength (Graham \& Heyer 1989). Taking into account that at increasing wavelengths the emission arises closer to the YSO, then the most reliable position of the source is from the $K \mathrm{~s}$ band: from the Zinnecker et al. (1999) image, the position of Re 4 IRS is $\alpha(\mathbf{J} 2000)=08^{\mathrm{h}} 20^{\mathrm{m}} 58.24$ and $\delta(\mathrm{J} 2000)=$ $-49^{\circ} 34^{\prime} 44^{\prime \prime} 6$ (with a position uncertainty of $\$ 0$ ' 3 : Correia, private communication).

Figure 6 shows that the near-IR emission associated with Re 4 IRS is not perfectly aligned with $\mathrm{HH} 188$ and the $\mathrm{H} \alpha$ knot 1: these two form a position angle of $149^{\circ}$, whereas the Ks emission peak of $\operatorname{Re} 4$ IRS has position angle of $158^{\circ}$ and $140^{\circ}$ with $\mathrm{HH} 188$ and the $\mathrm{H} \alpha$ knot 1 , respectively. This suggests that the powering source of the HH 188 system should be located roughly few arcsecs west of near-IR peak emission. A tentative position for the powering source can be obtained assuming that it is located equidistantly between $\mathrm{HH} 188$ and the $\mathrm{H} \alpha$ 1, that is: $\alpha(\mathbf{J} 2000)=08^{\mathrm{h}} 20^{\mathrm{m}} 57^{\mathrm{s}} .94$ and $\delta(\mathrm{J} 2000)=-49^{\circ} 34^{\prime} 44^{\prime \prime} .7$. This is $\sim 3^{\prime \prime}$ west of the $\operatorname{Re} 4$ IRS position derived previously from the Ks image. Table 3 shows the distance and position angle of the different $\mathrm{H} \alpha$ knots with respect to this tentative position: all the $\mathrm{H} \alpha$ knots are very well aligned with respect to this position with a position angle of $149^{\circ} \pm 1^{\circ}$. In addition, as can be seen in Fig. 6, one of the two jets detected by Zinnecker et al. (1999) coincides with $\mathrm{HH} 188$ and is also well aligned with the direction of the HH 188 system. Thus, as already suggested by Zinnecker et al. (1999), it seems that Re 4 IRS is a binary system. The protostar powering the HH 188 system is possibly a very embedded

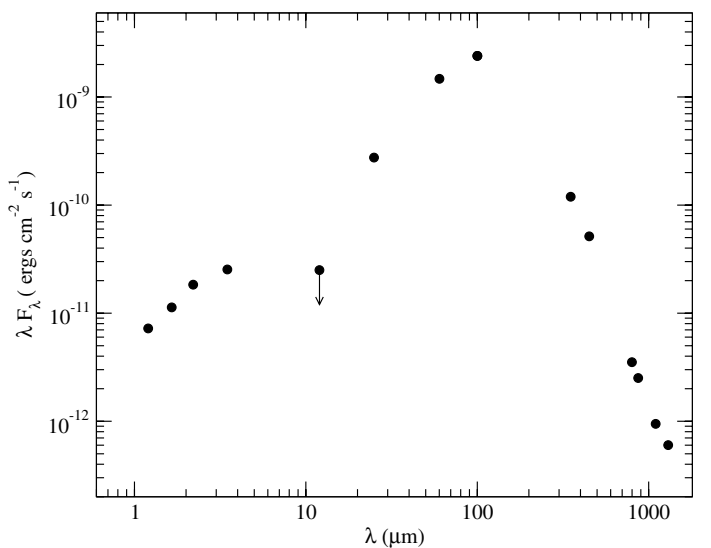

Fig. 5. Spectral energy distribution of Re 4 IRS, IRAS 08194-4925. Values at the $J H K L$ near-IR bands $(1.2,1.65,2.2$ and $3.4 \mu \mathrm{m}$, respectively) are from Graham (1986). The values from IRAS bands (12, 24, 60 and $100 \mu \mathrm{m}$ ) are from Cohen \& Schwartz (1987). Submm and $\mathrm{mm}$ values at $350,450,800$ and $1100 \mu \mathrm{m}$ are from Dent et al. (1998) and at 870 and $1300 \mu \mathrm{m}$ from Reipurth et al. (1993).

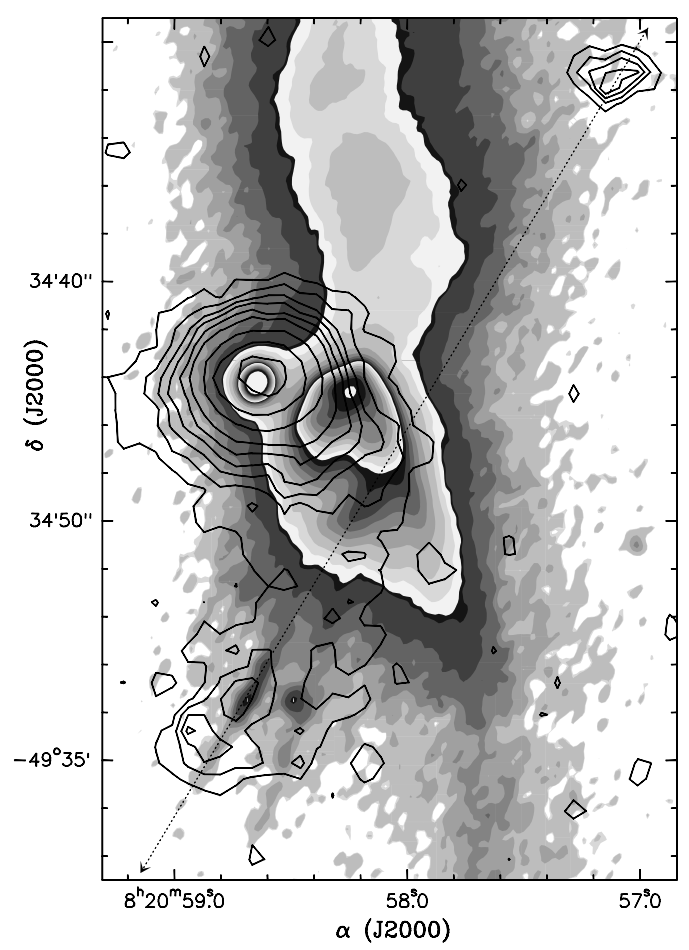

Fig. 6. Overlap of the contour $\mathrm{H} \alpha$ image from the SuperCOSMOS $\mathrm{H} \alpha$ survey (Parker et al. 2005) and the grey scale VLT Ks image from Zinnecker et al. (1999). In order to better show the two faint jets, the Ks image shown here was obtained by convolving the original VLT image with a Gaussian of $0.5 \times 0 .{ }^{\prime} 2$ and PA $=149^{\circ}$. The dotted line shows the direction of the proposed HH system associated with HH 188.

object, too faint in the near-IR to be detected. On the other hand, the protostar associated with the near-IR cometary-like emission could be the powering source of the other jet detected in the Ks image (see Fig. 6).

The different $\mathrm{H} \alpha$ knots aligned with $\mathrm{HH} 188$ and Re 4 IRS span over a length of $10^{\prime}$ or $1.2 \mathrm{pc}$ in projection. The two brightest spots are the two farthest and southernmost objects, 4 and 5 from Table 3. The properties of the HH 188 can be derived from the spectroscopical observations obtained by Graham (1986): Table 5 of that paper gives the relative intensities (with respect to 
$\mathrm{H} \alpha$ ) of the different emission lines detected ([SII], $\mathrm{H} \alpha$, [OI] and [NaII]). From the $\lambda 6716+\lambda 6730[\mathrm{SII}] / \mathrm{H} \alpha$ and [SII] $\lambda 6716 /[\mathrm{SII}]$ $\lambda 6730$ lines ratios we estimate that $\mathrm{HH} 188$ is a high excitation object with a high electron density $\left(n\left(\mathrm{e}^{-}\right) \simeq 2000 \mathrm{~cm}^{-3}\right)$. For the other knots further observations are required to characterize their properties.

\subsection{The origin of the $\mathrm{HCO}^{+}$emission}

The $\mathrm{HCO}^{+} 3-2$ peak is close to $\mathrm{HH} 188$ and offset by $\simeq 18^{\prime \prime}$ to the southeast with respect to the peak position of Ks emission from $\operatorname{Re} 4$ IRS. If, instead we use the tentative position of the HH 188 system's driving source, the offset is $\simeq 20^{\prime \prime}$. This offset between the $\mathrm{HCO}^{+}$emission and the protostar is comparable to the beam diameter of the $\mathrm{HCO}^{+}$map, so it is significative.

The fractional abundances derived in Sect. 3 assume that the emission comes from the central source, as we derived the $\mathrm{H}_{2}$ column density from the dust measurement at $1.3 \mathrm{~mm}$ that peaks at Re 4 IRS. However, the properties of the molecular emission that we see i.e. (i) a displacement to the southeast close to $\mathrm{HH} 188$, (ii) no significant velocity gradients, and (iii) narrow line widths, suggest that the molecular gas comes, at least partly, from an unperturbed dynamically or quiescent region. Nevertheless, because the angular resolution of our observations is not high enough to spatially resolve the IR and $\mathrm{HH}$ objects, we can not easily determine where the molecular emission comes from. Hence below we qualitatively investigate different possibilities.

At first we consider the most straightforward possibility that the emission comes from the dense circumstellar envelope around the protostar Re 4 IRS. If we assume that the SO and $\mathrm{HCO}^{+}$come from the cold, outer envelope around the protostar, then their abundances are a factor of $\sim 2$ respectively lower and higher with respect to the average value in the sample of Class 0 and I low mass protostars studied by Jørgensen et al. (2004), but still within the range of derived values. The $\mathrm{CH}_{3} \mathrm{OH}$ is expected to be significantly enhanced in the warm, inner envelopes $(T \gtrsim$ $90 \mathrm{~K}$ ) around low mass protostars (Maret et al. 2005; Jørgensen et al. 2005). Our observations of $\mathrm{CH}_{3} \mathrm{OH}$ around $\mathrm{Re} 4$ IRS yield an abundance higher than the range of values for the outer envelopes given by Maret et al. (2005) but much lower than the values found for the inner envelopes. The low $\mathrm{CH}_{3} \mathrm{OH}$ line width $\left(\simeq 0.6 \mathrm{~km} \mathrm{~s}^{-1}\right)$ and the non-detection of the $2_{0}-1_{0} \mathrm{E}$ transition in Re 4 IRS discards the inner envelope origin: in the Maret et al. (2005) sample, the line widths are always larger than $1 \mathrm{~km} \mathrm{~s}^{-1}$, with several cases where the line widths are few $\mathrm{km} \mathrm{s}^{-1}$, and the $2_{0}-1_{0}$ E transition should have been detected for densities higher than few times $10^{5} \mathrm{~cm}^{-3}$. Therefore, our observations suggests that a significant fraction of the $\mathrm{CH}_{3} \mathrm{OH}$ emission does not arise from the envelope around the IRAS protostar. In fact in other star forming regions, quiescent methanol emission has been detected significantly offset from the protostars and not too far from shocks (Palau et al. 2007; Girart, private communication).

These concerns led us to take into consideration alternative scenarios for the origin of at least a significant fraction of the observed molecular emission. It could be that most of the molecular emission arises from the interface along the HH 188 jet with the ambient cloud: in low-mass star forming regions, observations of enhanced $\mathrm{HCO}^{+}$toward several Class 0 objects can indeed be interpreted as coming from the walls of the outflow cavity (Hogerheijde et al. 1998; Rawlings et al. 2000; Redman et al. 2004). It is not unreasonable to assume that highly turbulent interfaces may be present in all environments where $\mathrm{HH}$ jets interact with the surrounding molecular cloud. Such interfaces have
Table 4. Abundance ratios ${ }^{a}$.

\begin{tabular}{lcc}
\hline \hline Region & $X[\mathrm{SO}] / X\left[\mathrm{HCO}^{+}\right]$ & $X\left[\mathrm{CH}_{3} \mathrm{OH}\right] / X\left[\mathrm{HCO}^{+}\right]$ \\
\hline HH 188 & 0.2 & 0.8 \\
HH 2 I1 & 0.2 & 1.2 \\
HH 34E & 0.9 & 1.5 \\
HH 1 & 0.8 & 2.1 \\
\hline
\end{tabular}

${ }^{a}$ The uncertainty of these ratios is approximately $30 \%$.

been shown to be characterized by a chemistry that is quite distinct in nature from that of typical dense cores and would not be achieved by any modification of conventional cold-cloud chemistry (Lim et al. 2001; Rawlings \& Hartquist 1997). However, if the emission were coming from an interface one would expect larger $\left(\geq 1 \mathrm{~km} \mathrm{~s}^{-1}\right)$ linewidths.

Another possibility is whether the emission arises from the cold, illuminated, gas ahead of the HH 188 object: quiescent, cold, molecular condensations have been found ahead of several $\mathrm{HH}$ objects in enhanced emission of $\mathrm{HCO}^{+}$(e.g. Rudolph \& Welch 1988; Torrelles et al. 1992; Girart et al. 2005) and can be explained as the result of photochemistry generated by UV radiation from the $\mathrm{HH}$ shock which leads to evaporation of icy mantles on dust grains in small density enhancements (e.g. Viti \& Williams 1999). In favour of this scenario it is important to point out that the $\lambda 6716+\lambda 6730[\mathrm{SII}] / \mathrm{H} \alpha$ lines ratio estimated in HH 188 is similar to the one derived for HH 34 (Morse et al. 1992). HH 34, as well as other high-excitation objects, is known to have apparently illuminated dense molecular gas. Recently Viti et al. (2006) presented a molecular survey ahead of several $\mathrm{HH}$ objects and found that the typical density, temperature, and line widths of such clumps are $10^{5}-10^{6} \mathrm{~cm}^{-2}, 10-20 \mathrm{~K}$, and $0.4-1.5 \mathrm{~km} \mathrm{~s}^{-1}$ respectively; the derived ranges of column densities for $\mathrm{HCO}^{+}, \mathrm{CH}_{3} \mathrm{OH}$ and $\mathrm{SO}$ were respectively: $5 \times 10^{12}-2 \times$ $10^{14}, 3 \times 10^{13}-4 \times 10^{14}$, and $8 \times 10^{12}-9 \times 10^{13} \mathrm{~cm}^{-2}$. The observed column densities around IRAS 08194-4925 are indeed consistent with these ranges. This together with the fact that the $\mathrm{HCO}^{+}$peak is displaced with respect to the IRS source and the line widths of the emission lines are of the same order as those from the molecular emission detected ahead of all the $\mathrm{HH}$ objects in the survey make this scenario a plausible one. It is then interesting to compare the ratio of column densities derived here with those from the objects in the survey by Viti et al. (2006) and with HH 2, whose complex interaction with its surroundings has been extensively studied (Girart et al. 2002; Viti et al. 2003; Lefloch et al. 2005; Girart et al. 2005). We list these ratios in Table 4. The reason for using ratios rather than individual column densities is to make the comparisons independent of the telescope. Both ratios indicate that if the molecular emission is indeed coming from the quiescent gas ahead of the $\mathrm{HH}$ object then it is closer in characteristics to HH 2 than $\mathrm{HH} 1$ and $\mathrm{HH}$ 34. The region surrounding HH 2 is very complex: for this comparison we chose to use the ratios derived from the densest (and closer to the HH 2) part of the region that Girart et al. (2005) interpret as being quiescent and directly illuminated by the UV coming from $\mathrm{HH} 2$ (measured to be $\sim 40$ Habing, see Molinari \& Noriega-Crespo 2002). Hence, if this scenario is correct, the gas we observe around HH 188 has a density of $\sim 10^{5} \mathrm{~cm}^{-3}$, and a temperature of $\sim 15 \mathrm{~K}$.

Finally, we consider the possibility that the background radiation from the Gum Nebula is the source of the UV radiation that is altering chemically the molecular gas in a similar way as the $\mathrm{HH}$ object. The Gum Nebula shows diffuse and extended $\mathrm{H} \alpha$ and radio emission, which suggests that it has also associated 
Table 5. Molecular line results for HH 188.

\begin{tabular}{|c|c|c|c|c|}
\hline $\begin{array}{l}\text { Molecular } \\
\text { transition }\end{array}$ & $\begin{array}{l}T_{\mathrm{mb}} \\
(\mathrm{K})\end{array}$ & $\begin{array}{r}\int T_{\mathrm{mb}} \mathrm{d} v \\
\left(\mathrm{~K} \mathrm{~km} \mathrm{~s}^{-1}\right)\end{array}$ & $\begin{array}{c}v_{\mathrm{LSR}} \\
\left(\mathrm{km} \mathrm{s}^{-1}\right)\end{array}$ & $\begin{array}{r}\Delta v_{\mathrm{LSR}} \\
\left(\mathrm{km} \mathrm{s}^{-1}\right)\end{array}$ \\
\hline \multicolumn{5}{|c|}{$\left(0^{\prime \prime}, 0^{\prime \prime}\right)$ offset position } \\
\hline $\mathrm{H}^{13} \mathrm{CO}^{+} 1-0$ & $0.80(2)$ & $0.59(2)$ & $3.79(1)$ & $0.70(3)$ \\
\hline $\mathrm{HCO}^{+} 3-2$ & $2.32(26)$ & $2.20(16)$ & $3.60(3)$ & $0.89(7)$ \\
\hline $\mathrm{CH}_{3} \mathrm{OH} 2_{0}-1_{0} \mathrm{~A}^{+}$ & $0.44(5)$ & $0.27(2)$ & $3.74(1)$ & $0.57(2)$ \\
\hline $\mathrm{CH}_{3} \mathrm{OH} 2_{-1}-1_{-1} \mathrm{E}$ & $0.44(5)$ & $0.27(2)$ & fixed $^{a}$ & fixed $^{a}$ \\
\hline $\mathrm{CH}_{3} \mathrm{OH} 2_{0}-1_{0} \mathrm{E}$ & $\lessgtr 0.12$ & & fixed $^{a}$ & fixed $^{a}$ \\
\hline $\mathrm{SO}_{5}-5_{4}$ & $0.81(5)$ & $0.69(3)$ & $3.64(1)$ & $0.81(4)$ \\
\hline \multicolumn{5}{|c|}{$\left(0^{\prime \prime},-20^{\prime \prime}\right)$ offset position } \\
\hline $\mathrm{H}^{13} \mathrm{CO}^{+} 1-0$ & $0.83(2)$ & $0.65(2)$ & $3.74(1)$ & $0.73(3)$ \\
\hline $\mathrm{HCO}^{+} 3-2$ & $2.43(22)$ & $2.12(13)$ & $3.52(3)$ & $0.82(6)$ \\
\hline \multicolumn{5}{|c|}{$\left(20^{\prime \prime},-20^{\prime \prime}\right)$ offset position } \\
\hline $\mathrm{H}^{13} \mathrm{CO}^{+} 1-0$ & $0.66(2)$ & $0.51(2)$ & $3.74(2)$ & $0.72(4)$ \\
\hline $\mathrm{HCO}^{+} 3-2$ & $1.82(28)$ & $2.00(18)$ & $3.63(5)$ & $1.03(10)$ \\
\hline \multicolumn{5}{|c|}{$\left(20^{\prime \prime}, 0^{\prime \prime}\right)$ offset position } \\
\hline $\mathrm{H}^{13} \mathrm{CO}^{+} 1-0$ & $0.60(4)$ & $0.51(3)$ & $3.85(2)$ & $0.80(4)$ \\
\hline $\mathrm{HCO}^{+} 3-2$ & $1.65(26)$ & $1.68(19)$ & $3.64(5)$ & $0.95(14)$ \\
\hline
\end{tabular}

${ }^{a}$ The three methanol lines were fitted simultaneously.

significant UV radiation generated by $\mathrm{OB}$ stars and/or by and old supernova remnant (e.g. Woermann et al. 2000). In order to consider this possibility we checked the SHASSA H $\alpha$ survey (Gaustad et al. 2001). The Sandqvist 109 molecular cloud (which includes the $\operatorname{Re} 4$ IRS region) is at a distance of $\sim 30^{\prime}$ from the brightest $\mathrm{H} \alpha$ emission regions of the Gum nebula. The background $\mathrm{H} \alpha$ emission around Re 4 IRS regions is about 2 to 3 times weaker than these bright regions. We have also checked the SHASSA survey around two other high excitation $\mathrm{HH}$ objects with irradiated dense molecular clumps, HH 2 and HH 34, both in the Orion star forming region, where there is also diffuse $\mathrm{H} \alpha$ emission. The level of diffuse $\mathrm{H} \alpha$ emission around these two objects is similar to the level in the Re 4 IRS region. HH 34 (Viti et al. 2006) and in particular HH 2 (Girart et al. 2005) exhibit molecular emission that has been succesfully modelled using the UV radiation-driven chemistry scenario. Although the possibility that the diffuse UV radiation contributes to the illumination of the molecular condensation can not be ruled out, the close spatial connection of the molecular emission with the $\mathrm{HH}$ objects indicates that the main source of $\mathrm{UV}$ radiation comes from the $\mathrm{HH}$ objects.

\section{Summary}

In this paper we presented maps of the region around Re 4 IRS in $\mathrm{HCO}^{+}(3-2)$ and $\mathrm{H}^{13} \mathrm{CO}^{+}(1-0)$, as well as observations in $\mathrm{SO}\left(6_{5}-5_{4}\right)$ and $\mathrm{CH}_{3} \mathrm{OH}\left(2_{0}-1_{0} \mathrm{~A}^{+}\right)$of the central position. We find that the $\mathrm{HCO}^{+}$emission is quite compact around the protostar but its peak is displaced to the southeast close to the HerbigHaro object HH 188. All the lines are narrow $\left(\sim 0.7 \mathrm{~km} \mathrm{~s}^{-1}\right)$ and $\mathrm{HCO}^{+}$does not show any sign of infall. Column densities were derived assuming LTE conditions.

We have also checked the SuperCosmos $\mathrm{H} \alpha$ survey and have found several knots of pure $\mathrm{H} \alpha$ emission very well aligned with HH 188 and forming an apparent parsec scale HH system. The driving source of this system is possibly an embedded protostar invisible in the near-IR. We tentatively estimate that it is located about $\sim 3^{\prime \prime}$ west of the Ks emission peak. The protostar illuminating the near-IR cometary-like nebula is possibly the exciting source of a faint jet. Thus, Re 4 IRS, a late Class 0 or Class I object, seems to be a binary system, as suggested by Zinnecker et al. (1999).
We attempt a very simple chemical analysis of the molecular emission in order to shed light on its origin. We consider four scenarios as the possible origin of the emission: the circumstellar envelope around the protostar; an interface along the HH 188 jet with the ambient cloud; the quiescent, cold, illuminated gas ahead of the $\mathrm{HH} 188$ jet; illumination by the diffuse Gum Nebula emission. The derived column densities, as well as the narrowness of the linewidths and the morphology of the $\mathrm{HCO}^{+}$emission are in agreement with the third scenario making it a plausible one, although it is still possible that there is contribution from the outer cold envelope around the protostar.

These observations have shown that the Re 4 IRS-HH 188 would make an interesting southern target for future ALMA studies of star forming regions.

Acknowledgements. We thank H. Zinnecker and S. Correia for providing the VLT Ks image. J.M.G. acknowledges support by MCyT grant AYA200508523-C03-02. S.V. acknowledges individual financial support from a PPARC Advanced Fellowship. J.M.G. and S.V. acknowledge support by a joined Royal Society and CSIC travel grant. This publication makes use of data products from the Two Micron All Sky Survey, which is a joint project of the University of Massachusetts and the Infrared Processing and Analysis Center/California Institute of Technology, funded by the National Aeronautics and Space Administration and the National Science Foundation.

\section{References}

Cohen, M., \& Schwartz, R. D. 1987, ApJ, 316, 311

Dent, W. R. F., Matthews, H. E., \& Ward-Thompson, D. 1998, MNRAS, 301, 1049

Gaustad, J. E., McCullough, P. R., Rosing, W., \& Van Buren, D. 2001, PASP, 113,1326

Girart, J. M., Viti, S., Williams, D. A., Estalella, R., \& Ho, P. T. P. 2002, A\&A, 388,1004

Girart, J. M., Viti, S., Estalella, R., \& Williams, D. A. 2005, A\&A, 439, 601

Graham, J. A. 1986, ApJ, 302, 352

Graham, J. A., \& Heyer, M. 1989, PASP, 101, 573

Hogerheijde, M. R., van Dishoeck, E. F., Blake, G. A., \& van Langevelde, H. J. 1998, ApJ, 502, 315

Jørgensen, J. K., Schöier, F. L., \& van Dishoeck, E. F. 2004, A\&A, 416, 603 Jørgensen, J. K., Schöier, F. L., \& van Dishoeck, E. F. 2005, A\&A, 437, 501 Langer, W. D., \& Penzias, A. A. 1993, ApJ, 408, 539

Lefloch, B., Cernicharo, J., Cabrit, S., \& Cesarsky, D. 2005, A\&A, 433, 217

Lim, A. J., Rawlings, J. M. C., \& Williams, D. A. 2001, A\&A, 376, 336

Maret, S., Ceccarelli, C., Tielens, A. G. G. M., et al. 2005, A\&A, 442, 527

Molinari, S., \& Noriega-Crespo, A. 2002, AJ, 123, 2010

Morse, J. A., Hartigan, P., Cecil, G., Raymond, J. C., \& Heathcote, S. 1992, ApJ, 399,231

Ossenkopf, V., \& Henning, T. 1994, A\&A, 291, 943

Palau, A., Estalella, R., Girart, J. M., et al. 2007, A\&A, 465, 219

Parker, Q. A., et al. 2005, MNRAS, 362, 689

Rawlings, J. M. C., \& Hartquist, T. W. 1997, ApJ, 487, 672

Rawlings, J. M. C., \& Yates, J. A. 2001, MNRAS, 326, 1423

Rawlings, J. M. C., Taylor, S. D., \& Williams, D. A. 2000, MNRAS, 313, 461

Redman, M. P., Rawlings, J. M. C., Yates, J. A., \& Williams, D. A. 2004, MNRAS, 352, 243

Reipurth, B. 1981, A\&AS, 44, 379

Reipurth, B., Chini, R., Krugel, E., Kreysa, E., \& Sievers, A. 1993, A\&A, 273, 221

Rudolph, A., \& Welch, W. J. 1988, ApJ, 326, L31

Sandqvist, A. 1977, A\&A, 57, 467

Torrelles, J. M., Rodriguez, L. F., Canto, J., et al. 1992, ApJ, 396, L95

Viti, S., \& Williams, D. A. 1999, MNRAS, 310, 517

Viti, S., Girart, J. M., Garrod, R., Williams, D. A., \& Estalella, R. 2003, A\&A, 399, 187

Viti, S., Girart, J. M., \& Hatchell, J. 2006, A\&A, 449, 1089

Whitney, B. A., Wood, K., Bjorkman, J. E., \& Cohen, M. 2003, ApJ, 598, 1079 Woermann, B., Gaylard, M. J., \& Otrupcek, R. 2000, MNRAS, 315, 241

Zhou, S., Evans, N. J., II, Koempe, C., \& Walmsley, C. M. 1993, ApJ, 404, 232 Zinnecker, H., Krabbe, A., McCaughrean, M. J., et al. 1999, A\&A, 352, L73 